\title{
EDITORIAL
}

nature

cell biology

\section{Sustainable databases}

\section{Although the flood of cell biological knowledge rises relentlessly, many databases face an uncertain future. Unless funding for essential bioinformatic resources is set in stone, the next storm may wash away the foundation of future cell biology research.}

Many cell biologists are warming to Systems biology. Nevertheless, it's adoption is more a creeping cultural shift than a revolution. One reason is that biologists need time to learn the tools, or at least to collaborate with sympathetic physical scientists. The main limiting factor, however, is assembling datasets that are sufficiently broad and quantitative for meaningful systems level-interrogation. This requires databases that archive biological data and knowledge in a comprehensive, standardized, well-annotated and machine-readable form. Although databases such as the 'molecule pages' (www.signalling-gateway.org/molecule) are beginning to capture structured data to a significant level of depth, the published paper is nowhere near obsolete and it is therefore equally important to develop text-mining tools to query the literature directly.

\section{Opening up to text mining}

Broad access to the primary scientific literature is essential for anyone with serious research ambitions. However, publication of a thoroughly peer reviewed and edited paper is expensive. Much has been said about whether this should be financed by the reader or the author - either way, payment ultimately derives from research funding. From a systems viewpoint, it is only accessibility to machine-based interrogation that is important. This is guaranteed with open-access publication, but it need not be an issue for 'subscriber pays' models either: Nature is currently testing the Open TextMining Interface (OTMI), which scrambles text to make it unpalatable to the human eye, while largely conserving semantic relationships.

PubMed Central was set up by NCBI to provide a centralized long-term open-access repository for peer-reviewed information. A European counterpart, UKPMC, will be launched in January by a UK based consortium that includes EBI. Such platforms aid text mining and UKPMC is aiming to integrate the literature with databases using EBIs bioinformatic tools. A number of funding agencies are supporting these projects and require data deposition by grantees, although the impact on the financing of the publication process remains ill defined. Funding databases

The challenge to standardize and collect all meaningful data into public repositories has spawned a significant number of databases. Some, such as the International Nucleotide Sequence Databases (INSD) and UniProt kowledgebase (UniProtKB) hosted by the European Bioinformatics Institute(EBI), or NCBI's PubMed, are in every cell biologists basic toolbox. Key resources of the EBI and NCBI are described in more detail in last month's focus, Systems biology: a user's guide. Although research would rapidly cease without these bioinformatic powerhouses, it is important to emphasize that there are several hundred other databases out there, many of which provide invaluable resources to specialist communities and have set community standards, despite being maintained by insecure limited budgets. Some of these resources are under persistent threat of closure - a recent prominent example being the Schizosaccharomyces pombe community resource GeneDB - , whereas others, such as the Yeast Protein Database (now Biobase) have been commercialized.

Almost invariably, traditional funding sources are used to maintain these databases. It is relatively easy to obtain start-up funding for a new database in a promising niche. The real problem comes in maintaining a database after the first tenure of a grant expires. Databases are only meaningful if their content and bioinformatics are kept up-todate. Database maintenance is expensive - the Jackson labs Mouse Genome Database (MGD) costs \$4 million annually - and even the EBI has endured several financing shortfalls. The institute is financed principally through EMBL, the European Union, NIH and the Wellcome trust. Despite a commitment from EMBL to maintain the core databases, most of its resources rely on time-limited funding: FELICS, a project to interlink a number of principal European data resources, was recently launched with five years of funding and the long standing EMBOSS suite of bioinformatics tools struggled to receive another three year funding lifeline.

Of course, databases should be evaluated for their quality and utility to the community regularly - in fact just as regularly as research projects. However, different criteria must apply: research grants are principally evaluated on the basis of being timely, novel and achievable. Databases should be required to demonstrate that their content is up-to-date and comprehensive, that their architecture maximizes information access and ultimately that they are used by the community. The time has come to dissociate the funding of databases from research project grants. Research funding is limited and there is undoubtedly an overproliferation of databases with unnecessary redundancy. However, everyone would be best served if databases competed against one another rather than with primary research. Furthermore, once a database or a bioinformatic tool has become an essential community resource, its funding should be guaranteed indefinitely. Its performance should be monitored by independent evaluation with executive power to implement improvements. Importantly, survival of the resource must not be dependent on year-on-year re-evaluation.

Evaluating existing public bioinformatic resources in competition with hypothesis driven research makes no sense - it is like comparing apples and oranges, with the difference that one is a fruit ripe for the plucking and one a tree essential to grow and to sustain that fruit. The EC's $7^{\text {th }}$ framework programme (FP7) is an excellent opportunity to earmark separate funds to fertilize the tree in a sustainable manner.

Further reading on http://www.connotea.org/user/bpulverer/tag/database\%20funding 